\title{
Memory for targets in a multilevel simulated environment: Evidence for vertical asymmetry in spatial memory
}

\author{
PAUL N. WILSON \\ University of Leicester, Leicester, England \\ NIGEL FOREMAN \\ Middlesex University, London, England \\ DANAË STANTON \\ University of Nottingham, Nottingham, England \\ and \\ HESTER DUFFY \\ University of Leicester, Leicester, England
}

\begin{abstract}
In two experiments, adult participants explored a symmetrical three-tiered computer-simulated building that contained six distinctive objects, two on each floor. Following exploration, the objects were removed, and the participants were asked to make direction judgments from each floor, indicating the former positions of the objects on that floor and on higher and lower floors. Relative tilt error scores indicated a bias, in that targets that were higher than the test location were judged as consistently lower than their actual positions and targets that were lower than the test location were judged as consistently higher than their actual positions. Absolute tilt errors revealed an asymmetry, with more accurate and less variable tilt errors for judgments directed to lower floors than for judgments directed to higher floors. Experiment 3 ruled out an account of the findings that does not relate them to spatial memory. The results suggest that the superiority of downward over upward spatial judgments, previously reported in two-dimensional visual-spatial tasks, extends to navigational spatial memory.
\end{abstract}

When encoding spatial relationships, people typically adopt a frame of reference that is determined by the characteristics of the environment and their exposure to it. An important distinction has been made between allocentric and egocentric reference frames. Allocentric encoding occurs when spatial relationships are processed with respect to objects outside the observer, such as cardinal directions, or with respect to landmarks. Egocentric reference frames establish location with respect to the observer, such as retinal or body coordinates. Egocentric spatial information appears to be based on a coordinate system organized around extensions of the three major body axes: head/feet, front/back, and left/right. For example, Bryant, Tversky, and Franklin (1992) described scenes textually in which objects were located to the front, back, left, right, above, and below the reader's imagined position; when asked to retrieve the names of the objects, people were fastest for

The authors acknowledge financial support from Scope and British Telecom. We are grateful to Michael Tlauka and Diane Wildbur for helpful comments on an earlier version of the article. Correspondence concerning this article should be addressed to P. N. Wilson, Department of Psychology, University of Hull, Cottingham Road, Hull HU6 7RX, England (e-mail: p.wilson@ac.uk). those objects located along the head/feet axis, slower for the front/back axis, and slowest for the left/right axis. The authors suggested that the dominance of the head/feet axis is due to its correlation with the asymmetry in the axis of gravity in the real world; front/back is dominant over left/right, since it is correlated with an asymmetric body axis that depends on the forward dominance of the senses; the left/right axis is the least dominant, since it is symmetrical with respect to the world and the body.

Interestingly, Bryant et al. (1992, Experiment 1) found that after participants had read a description of a threedimensional array of objects, they were faster to respond from memory to the location of a test object if it had been described as below, rather than above, a referent object. Similar but small and nonsignificant differences were shown with other experiments (e.g., Bryant \& Tversky, 1999). Although not a consistent outcome, it is possible that Bryant et al.'s finding of faster responding to lower objects reflects an advantage to downward processing, possibly due to the effects of gravity. The possibility that the vertical dimension may be encoded asymmetrically has not received much experimental attention; the majority of spatial-learning studies have involved primarily the horizontal axis. However, there is some evidence sug- 
gesting that the processing of spatial information in the vertical dimension may not be symmetrical. For example, figure segmentation into figure and ground is more efficient when stimuli are presented in lower visual quadrants (Rubin, Nakayama, \& Shapley, 1996), and contralesional neglect is most pronounced in relation to stimuli located below the horizontal meridian (Ladavas, Carletti, $\&$ Gori, 1994). The functional separation of visual processing in upper and lower regions of visual space has been demonstrated using computer-generated three-dimensional virtual environments (VEs; Aguirre \& D'Esposito, 1997; Shelton \& Gabrieli, 2002). Recently, the lower visual field superiority has also been extended to a spatial memory task by Genzano, Di Nocera, and Ferlazzo (2001), who found that participants were better able to remember the locations of patterned blocks when they had appeared in the lower, as opposed to the upper, visual field during an exposure phase and also that this ability was adversely affected by spatial interference (finger tapping in a spatial sequence), but not by visual interference.

In the experiments reported in this article, the possibility of vertical asymmetry in spatial encoding was investigated, using computer-simulated VEs. VEs have the advantage that they can be tailored to the requirements of a particular experimental task and can be manipulated without the restrictions of real-world constraints (Wilson, 1997). Numerous studies in which VEs have been used have demonstrated that information about spatial locations of objects, their relative positions, and the routes that connect them can be effectively acquired from virtual exploration alone and that such information can be readily transferred to equivalent real environments by children and adults (Foreman, Stanton, Wilson, \& Duffy, 2003; Rossano, West, Robertson, Wayne, \& Chase, 1999; Stanton, Wilson, \& Foreman, 1996; Wilson, Foreman, \& Tlauka, 1997; see Péruch \& Gaunet, 1998, and Rose \& Foreman, 1999, for reviews). As in realworld studies, in most previous studies of VE exploration, the horizontal plane has been investigated, with movements (viewpoint shifts) through the virtual world being restricted to left-right rotation, and forward-back translation (e.g., Péruch, Vercher, \& Gauthier, 1995). Where multitiered environments have been simulated, the results of spatial testing have usually involved measures of route learning and have been mixed. Regian, Shebilske, and Monk (1992) asked participants to navigate through a virtual building with three floors, each containing four rooms; following practice, many were subsequently able to take novel routes through the building with maximum economy, completing routes that traversed the minimum number of rooms and taking paths that were significantly shorter than would occur by random choice. Witmer, Bailey, Knerr, and Parsons (1996) asked participants to take complex routes through three floors of a building, leading to six destinations in two office suites. Although the routes were well learned, configurational knowledge (measured via distance and direction estimates) showed no improvement. Richardson,
Montello, and Hegarty (1999), using a simulated building with two stories, reported poorer performance for testing on higher and lower floors than for testing on the same floor, and they suggested that disorientation arose from rotational movements required in stair areas connecting the levels. Difficulty in making judgments on different levels in VEs may reflect the fact that a multilevel environment is another form of large-scale environment, in which disorientation has been observed in VE participants (cf. Darken \& Sibert, 1996). The visual discontinuity (Kuipers, 1978; Poucet, 1993) between levels might make this kind of environment particularly difficult to remember from VE navigation alone. In the context of a possible asymmetry in spatial learning, it is interesting that, using a three-tiered computer-generated VE, Wilson, Foreman, and Tlauka (1997) found that when participants were asked to make pointing judgments at ground floor level toward targets on the same level, VE and real-exploration groups were equally accurate but that the horizontal component of pointing estimates of the VE group were less accurate than those of the real-exploration group when the targets were on a lower floor. Moreover, when pointing to targets on the floor above (the first floor), the VE group performed no better than controls, with both groups performing less accurately than the real-exploration group. The authors suggested that this asymmetry might have arisen from a poor representation of the stair area linking ground and upper levels in the VE. However, it is possible that this asymmetry in pointing accuracy reflects a more general bias for encoding location selow, rather than above, the test location.

In the present experiments, we examined both horizontal and vertical direction-estimating judgments in a simplified three-level VE that contained multiple targets. We were particularly interested in comparing the speed and the accuracy of judgments between remembered targets above and below the VE explorer, on the hypothesis that these measures might reflect differences in spatial memory.

\section{EXPERIMENT 1}

\section{Method}

Participants. The 27 participants were recruited from two sources. Five were clerical staff from the Nottingham office of Scope, and 22 were undergraduate and postgraduate students and research staff from the psychology department at Leicester University. Their ages ranged from 18 to 53 years (mean age, 25 years and 2 months), and 2 were male; all had normal or corrected-to-normal vision.

Apparatus. A Pentium III personal computer with a 21-in. monitor was used to display VEs created using the Superscape Virtual Reality Toolkit. Movement through the VE was effected by pressing the cursor keys on the keyboard: The up and down cursors effected forward and backward movements, whereas the left and right cursors effected left and right rotation.

Two multilevel VEs were created: the main experimental VE and a simple practice VE. The main experimental environment comprised three vertically aligned rooms that formed a three-story building (see Figure 1). In the software's arbitrary scale, the rooms 


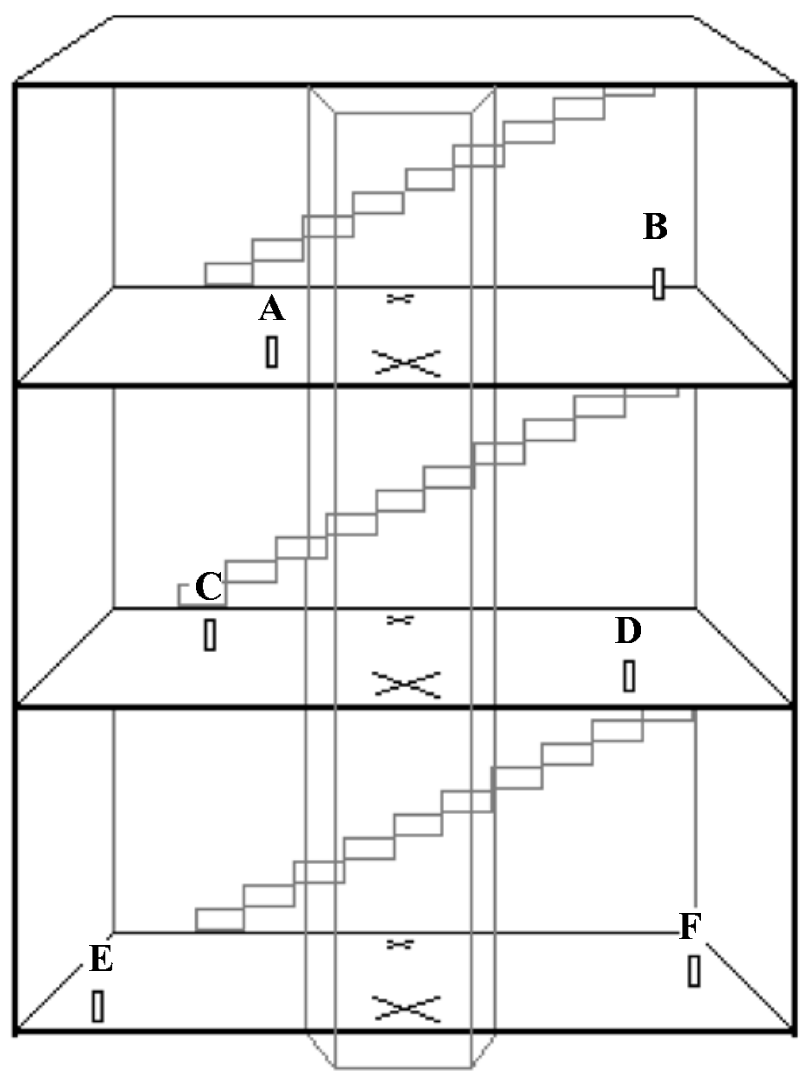

Figure 1. Illustration of the three-tiered virtual environment used in the main part of the experiment, showing example positions of the six target objects (A-F), the test locations (marked with crosses), the position of the stairs against the far wall, and an outline of the elevator shaft against the near wall.

were 100 units across, 78 units deep, and 36 units high. Assuming average human eye level to be $170 \mathrm{~cm}, 12$ units would equal approximately $1 \mathrm{~m}$. Therefore, subjectively, the rooms were approximately $8.3 \times 6.5 \times 3 \mathrm{~m}$; this equivalent meter scale is used in the descriptions that follow to give an intuitive measure. The floor and ceiling of each room were multicolored checkered texture-mapped patterns with different predominant colors in each room: The ceiling and floor of the lowest room were predominantly green; in the middle room, they were predominantly pink; and in the highest room, they were predominantly blue. In the middle of one of the longer walls on each floor was an open elevator shaft, $1.2 \times 1.6 \mathrm{~m}$ wide, which connected all three rooms. A working elevator comprised a vertically moving platform controlled by two buttons, labeled with an arrow pointing either up or down. Clicking on one of these buttons with the mouse cursor while inside the elevator controlled movement between floors. The elevator was open, so that if the participants were facing out toward the rooms, they could observe their movement between floors. On the wall of each room, next to the elevator shaft, there was a button labeled CALL, which, when activated by the mouse, brought the elevator to the appropriate floor.

Adjacent to the wall opposite to the elevator were centrally located staircases that connected the rooms (a floor-to-ceiling flight of stairs was also included on the top floor in order to equate the appearance of the rooms). Six target objects, two located in each room, were mounted on stands of slightly differing heights. The objects were the following: a globe with a diameter of $0.68 \mathrm{~m}$, colored blue and green, which continuously rotated on its own axis; a toy boat, $0.15 \times 0.70 \times 0.36 \mathrm{~m}$ high, which made a foghorn noise when activated with the mouse; a blue toy robot, $0.25 \times 0.2 \times 0.68 \mathrm{~m}$ tall, which continuously walked around in a circle on the spot; a red toy car, $0.8 \times 0.44 \times 0.35 \mathrm{~m}$ high, which made a car horn noise when activated with the mouse; a red flower with a green stem and leaves, $0.38 \times 0.22 \times 0.7 \mathrm{~m}$ high; and a toy train, predominantly gray and dark green, $0.71 \times 0.2 \times 0.37 \mathrm{~m}$ high, which made a train whistle sound when activated with the mouse. The relative locations of the objects in the different rooms were counterbalanced across participants. Each floor contained one test object that made a noise and one that did not. The height of the stands were adjusted so that the vertical angles to the center of the objects were approximately equated when estimations were made up or down between floors: The average height was $0.17 \mathrm{~m}$ on the top floor, $0.5 \mathrm{~m}$ on the middle floor, and $0.75 \mathrm{~m}$ on the lower floor.

For the test phase, all the objects and their stands were removed. Six static viewpoints were created: These were two central viewpoints on each floor, on opposite sides of the room, in the same relative locations (see Figure 1). The locations when one was facing from the elevator toward the stairs were $4 \mathrm{~m}$ from the left-hand wall and $1 \mathrm{~m}$ in from the facing (stairs) wall, and $4 \mathrm{~m}$ from the left-hand wall and $0.5 \mathrm{~m}$ from the near (elevator) wall. The cursor keys were used to control pointing, using cross hairs in the center of the screen: Pressing the left and right cursors effected rotation movements, and the up and down cursors effected tilt up and down (translation movements were disabled). Precise angles of tilt and rotation (to $1^{\circ}$ ) were displayed on an instrument panel at the side of the screen. The latencies taken to make judgments were also recorded.

The practice environment comprised a ground-level open square $(42 \times 42 \mathrm{~m})$, above one corner of which was suspended an open $12 \times 12 \mathrm{~m}$ square platform raised to a height of $5 \mathrm{~m}$. The platform could be accessed on one side via a flight of 17 stairs or directly opposite via an elevator; the elevator was very similar to that used in the main test environment. The ground, stairs, elevator exterior, and platform were colored a uniform brown and were set in an open featureless green space. The VE contained two practice test objects: A model sailboat, $0.9 \times 0.8 \mathrm{~m}$, was situated, off-center, on top of the platform, and a 1.2-m-square doll's house stood almost directly below it on the ground.

Procedure. The participants were tested individually in quiet rooms and were seated approximately $0.5 \mathrm{~m}$ from the computer screen. The practice VE was presented before the main experimental VE; the participants were encouraged to explore both levels freely, using both the stairs and the elevator. They were asked to take note of the locations of the sailboat and the doll's house and were asked to point toward these objects once they had been seen, but at locations from which the objects were not directly visible. Verbal feedback was given to correct these estimates. No time limit was imposed on this preliminary exploration, and the participants were asked to continue to explore until they felt confident using the keyboard keys to effect exploration. At the end of this phase, no participant reported or was observed to experience any diff iculty in exploring.

For the main part of the experiment, all the participants were initially presented with a view in the middle room, from just in front of the elevator facing toward the center of the room. The two objects in each room were illustrated, and the participants were told that some of these objects would react if activated with the mouse. They were told that they should explore all three rooms freely and for as long as they wished but that they should visit each room at least twice. The experimenter suggested that they could use both the elevator and the stairs to travel between floors, and the majority used both routes. The participants were also told that the purpose of this exploration was to remember the location of the six objects and that they would later be asked to identify these locations as they had done for the objects in the pretraining phase. 
When a participant stated that he or she was familiar with the objects and their locations, the test was started. The participants were told that they were looking at the same environment that they had explored, but with the objects removed. The new functions for the up and down keys were illustrated, and they were told that on some test trials, they would be required to point up through the ceiling or down through the floor to illustrate the former locations of some of the objects. All the participants were tested at each of the six test locations, and from each location, they were asked to point toward the former location of each of the six test objects in turn. At each change of test location, the experimenter verbally described the new location-for example, "You are now just in front of the lift [elevator], on the middle floor, facing toward the stairs." The six test locations were presented in spatially ascending order for half the participants, whereas for the remaining participants, they were presented in a spatially descending order. The order of testing the former object positions followed a pseudorandom pattern.

The time to make each judgment was recorded from the last word of the instructions to when the participant indicated that he or she had completed his or her judgment. For each judgment, the tilt and rotation angles were recorded.

\section{Results and Discussion}

An alpha level of $p<.05$ was adopted for all the analyses reported in this article. The sex of the participant was not included as a factor in any of the analyses, because there were too few male participants to make comparisons meaningful.

Error scores for the tilt and rotation components for each judgment were calculated separately by subtracting the angles of the participant's estimate of the directions to the target objects from the vertical and the horizontal components of the actual vectors. These error data were analyzed in two forms. First, the relative errors (retaining the direction of the error) were analyzed to see whether there were systematic biases in the direction of participants' estimates. Second, absolute errors (ignoring the direction of the error) were analyzed to provide an assessment of overall accuracy when the signs of the errors did not attenuate the mean errors.

Initially, the data were collated for each of five sets of measures (i.e., latencies to make the direction judgments, the relative and absolute rotation errors, and the relative and absolute tilt errors) by averaging, in each case, to provide a single score for two floors up, one floor up, the same floor, one floor down, and two floors down. These scores comprised the mean of 4 observations for two floors up and down, the mean of 8 observations for one floor up and down, and the mean of 12 observations for the same floor. However, inspection of the between-floor variability for the five data sets suggested that, although the variance in the response time and relative rotation scores was homogeneous, for the relative tilt, the absolute rotation, and absolute tilt scores, the data were heterogeneous. Heterogeneity of variance could be a consequence of the different numbers of observations that were averaged to provide mean scores for the different floors. Therefore, mean scores were derived from 4 observations from each floor, to equate the numbers of observations on the same floor and one floor up and down, with those available for two floors up and down. The raw scores were selected according to a pseudorandom process, with the constraint that, across participants, similar numbers of observations were taken from both sides of the relevant floors (indicated by crosses on the relevant floors in Figure 1).

Between-conditions variability was assessed by analyzing the squared deviations of the mean scores for each participant on each floor; the variances for each data set are presented in the top portion of Table 1. Heterogeneity was assessed in a separate within-subjects analysis of variance on the squared deviations, and significant effects are indicated in the right-hand columns of Table 1. Analyses showed significant differences for relative and absolute tilt scores, which as can be seen in Table 1, reflects greater variability for judgments on higher floors, with higher variance for two floors up than for one floor up; the variance on the lower floors was similar to that on the same floor. This analysis suggests that the vertical component to the participants' memories of upward directions was subject to greater variability than was that to downward directions.

Table 1

Variance Scores for the Five Measures Analyzed in Experiments 1 and 2

\begin{tabular}{|c|c|c|c|c|c|c|}
\hline \multirow[b]{2}{*}{ Measure } & \multicolumn{5}{|c|}{ Floor } & \multirow[b]{2}{*}{ Significance } \\
\hline & Two Down & One Down & Same & One Up & Two Up & \\
\hline \multicolumn{7}{|c|}{ Experiment 1} \\
\hline Time & 29 & 31 & 44 & 113 & 146 & n.s. \\
\hline Relative rotation & 302 & 166 & 241 & 315 & 308 & n.s. \\
\hline Relative tilt & 199 & 110 & 139 & 602 & 1,018 & $*$ \\
\hline Absolute rotation & 340 & 244 & 377 & 352 & 369 & n.s. \\
\hline Absolute tilt & 116 & 56 & 64 & 404 & 798 & $*$ \\
\hline \multicolumn{7}{|c|}{ Experiment 2} \\
\hline Time & 13.8 & 2.3 & 81.4 & 16.8 & 9.7 & $*$ \\
\hline Relative rotation & 91 & 50 & 393 & 110 & 141 & n.s. \\
\hline Relative tilt & 156 & 308 & 246 & 530 & 548 & n.s. \\
\hline Absolute rotation & 168 & 32 & 178 & 101 & 187 & n.s. \\
\hline Absolute tilt & 88 & 151 & 142 & 453 & 520 & $*$ \\
\hline
\end{tabular}

Note-n.s. indicates that the squared deviation scores do not differ significantly between floor levels; *indicates a statistically significant heterogeneity. 
Due to the heterogeneity of some data sets, nonparametric analyses of the differences between the data (i.e., the means of the selected scores) for each floor were undertaken: For each set of scores, a Friedman's rank test $(N=27, d f=4)$ was used to determine whether any overall differences between floor conditions was present; where significant differences were indicated, upper and lower floor judgment scores were compared, using the Wilcoxon signed-ranks test on the averaged scores for one and two floors up, as compared with the averaged scores for one and two floors down.

The mean latencies to make judgments are illustrated in the top panel of Figure 2. An analysis of these data showed no significant difference between conditions $\left(\chi^{2}=4.0, p>.05\right)$.

The mean relative rotation errors are displayed in the middle panel of Figure 2. Negative error scores represent errors made toward the left of the target, and positive errors
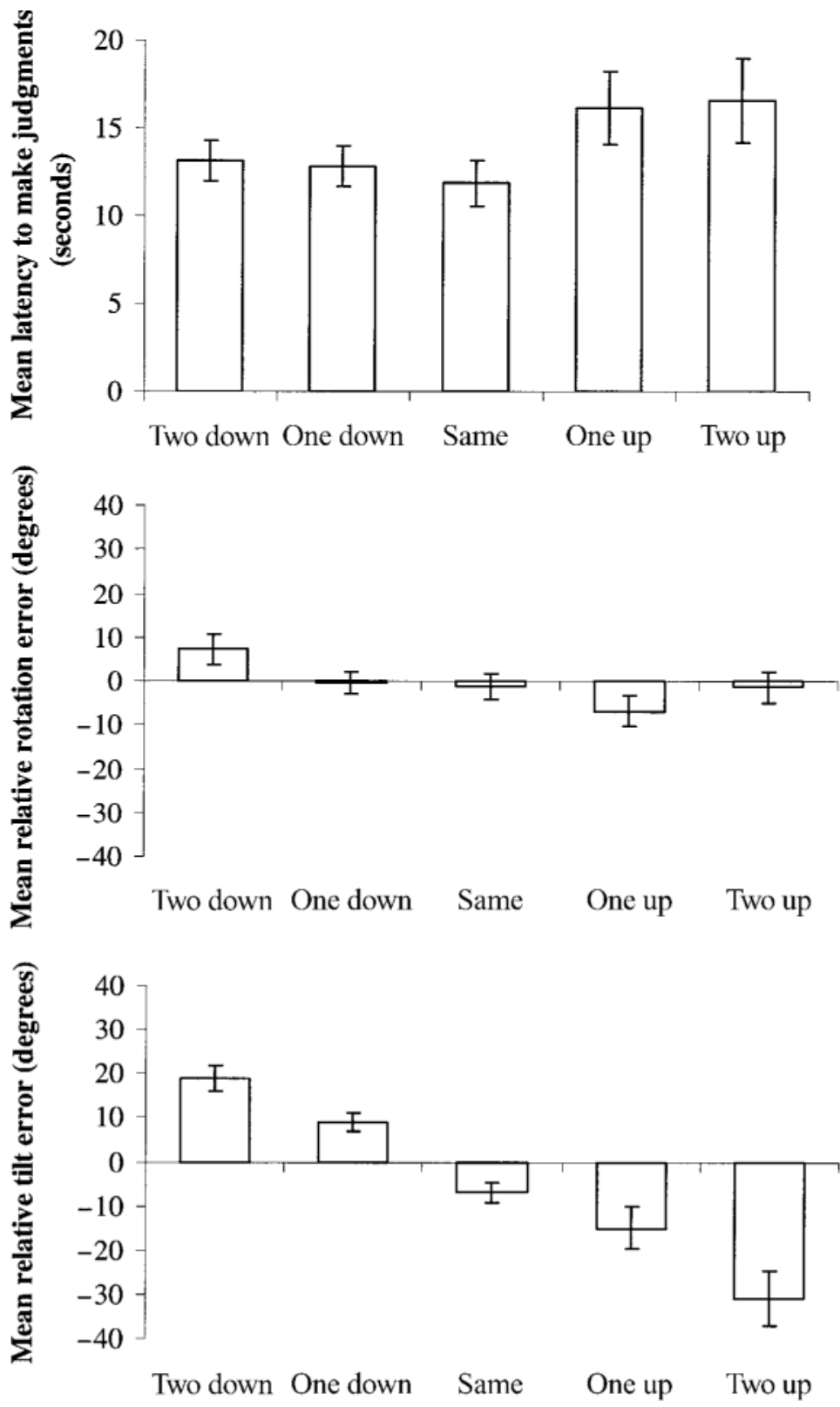

Figure 2. Results of Experiment 1. The top panel illustrates the mean latencies to make the tilt and rotation judgments about the former position of objects that were two floors up or down, one floor up or down, or on the same floor. The middle panel illustrates the mean relative rotation errors for these judgments. The bottom panel illustrates the mean relative tilt errors for the same judgments. The error bars represent one estimated standard error above and below the mean. 
represent errors made to the right of the target. An analysis of these data showed no significant difference between means $\left(\chi^{2}=8.03, p>.05\right)$. Since relative rotation errors could reveal a bias to make judgments to the left or the right, it is important to know whether there is a significant bias in either direction in the data from the same floor, when tilt is minimally involved in the judgment; no significant difference was apparent between the number of left- and right-biased judgments: 10 of the 27 judgments tended to the right (binomial probability $>.1$ ).

The mean relative tilt errors are illustrated in the bottom panel of Figure 2. Negative scores represent judgments on which the participant estimated the target to be lower than it actually was, whereas positive scores represent estimates on which the participant judged the target to be higher than it actually was. An analysis revealed a significant overall difference between floors $\left(\chi^{2}=51.9\right.$, $p<.05)$, with a significant difference between upward and downward judgments $(z=4.13, p<.05)$, errors on upward judgments were biased toward lower estimates, and errors on downward judgments were biased toward higher estimates. The relative tilt errors on the same floor were analyzed separately, and this analysis suggested a bias, with 20 of the 27 participants making judgment errors below the horizontal (binomial probability $<.05$ ).

The mean absolute rotation errors are presented in the upper panel of Figure 3. An analysis revealed a significant overall difference between floors $\left(\chi^{2}=17.8, p<\right.$ $.05)$, with significantly less accurate upward than downward judgments $(z=2.62, p<.05)$.

The mean absolute tilt errors are presented in the lower panel of Figure 3. Analysis revealed a significant overall difference between floors $\left(\chi^{2}=38.4, p<.05\right)$, with significantly less accurate upward than downward judgments $(z=2.51, p<.05)$.

Combining the data for each participant within each floor could conceivably obscure systematic effects based on the magnitude of the vectors to different objects, particularly given the differences in variation between judgments on different floors. Therefore, the data for individual object locations were collated with respect to their degree of rotation and tilt from each of the test viewpoints.
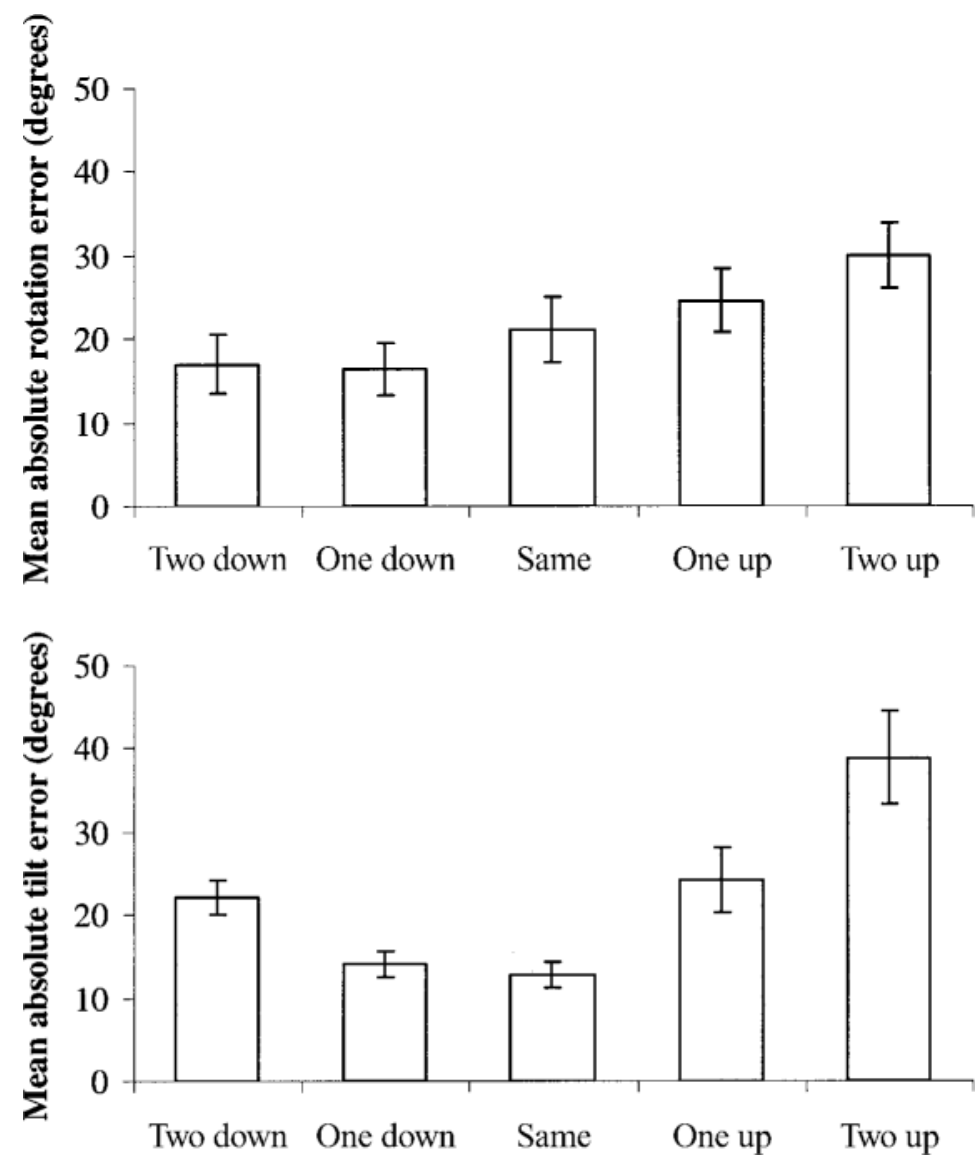

Figure 3. Results of Experiment 1. The top panel illustrates the mean absolute rotation errors for judgments of the former position of objects that were two floors up or down, one floor up or down, or on the same floor. The bottom panel illustrates the mean absolute tilt errors for these judgments. The error bars represent one estimated standard error above and below the mean. 
Across the counterbalancing of the locations of the six objects and six test locations, this reorganization of the data provided 39 different rotation measures (range, $85^{\circ}$ to $-87^{\circ}$ ) and 45 different tilt measures (range, $64^{\circ}$ to $-63^{\circ}$ ). Mean scores for each object location from each test site were derived from the data of the subgroups of participants that counterbalanced location testing. These data, which comprise 72 tilt and rotation observations, were collated for measures of response time, relative rotation errors, and relative tilt errors.

Spearman's rho correlation analyses $(N=72$ in each case) on the relative tilt and rotation error measures showed a medium-sized negative correlation between the actual degree of rotation and the participants' relative errors in judgments of rotation $(r=-.40, p<.05$; see the top panel of Figure 4 ) and a large negative correlation between the actual degree of tilt and the participants' relative tilt judgments $(r=-.84, p<.05$; see the bottom panel of Figure 4). In both cases, the correlations reflect estimates that increase in bias toward the center point (i.e., directly ahead) as the actual angles to the object locations increase from the center point, both horizontally (actual rotation) and vertically (actual tilt). The significant rotation correlation, although not particularly large, revealed an effect that was not significant in the combined floor data (middle panel of Figure 2): a tendency to make right errors to objects to the left and left errors on objects to the right. The significant tilt correlation supports the effect reported in the combined floor data for each participant (see the lower panel of Figure 2) and confirms that the downward bias on upward judgments is greater than the upward bias on downward judgments. Analyses of the response time data did not show significant correlations with either the degree of actual rotation or the degree of actual tilt.

The main findings of interest in Experiment 1 were the following. First, relative tilt errors displayed a consistent and substantial bias, with mean judgments on both higher and lower floors biased toward the horizontal; that is, when judging the position of objects on higher floors, the participants made errors with a predominantly downward bias toward the horizontal view, whereas judgments on lower floors were predominantly biased upward toward the horizontal view. This biasing effect increased as the angle to the test objects increased from the horizontal ahead view. Second, the magnitude and variability of the tilt errors was greater for upward than for downward judgments; this effect appears to have been due primarily to the downward bias on upward judgments ( $M$ of one and two floors up $=-23.2^{\circ}$ ) being larger than the upward bias on downward judgments ( $M$ of one and two floors down $\left.=14.8^{\circ}\right)$. Interestingly, the participants displayed a downward memory bias in relative tilt errors on the same floor. These data are among the first to suggest vertical asymmetry in spatial memories, with a bias in favor of memories for locations that are on a lower, rather than a higher, plane.

The greater accuracy and lower variation of downward than of upward judgments might not appear to be too surprising, given the studies that have shown visual field asymmetry (see the introduction): We live in a world in which we interact more frequently with objects that are below eye level. When walking, we pay more attention to the ground than we do to the sky in order to maintain sure footing; inside buildings, we usually see desks, chairs, cupboards, and so on, below eye level; when we sit at a desk, our computers, papers, and other items rest on the table top below eye level. In general, lower regions of visual space are relatively close to the perceiver (i.e., peripersonal space) and are of special practical significance in terms of reaching movements toward targets and obstacle avoidance. The upper visual field is more likely to support distal stimulus processing (i.e., extrapersonal space; see Previc, 1990, 1998). Therefore, in accordance with the central ideas of the spatial framework analysis (Bryant \& Tversky, 1999; Bryant et al., 1992; Franklin \& Tversky, 1990), the effects of gravity may ensure that, at least under some circumstances, downward spatial processing is dominant.

\section{EXPERIMENT 2}

Although the vertical asymmetry noted in Experiment 1 may be accounted for by appealing to prior learning experiences (which does not exclude the possibility of an innate bias), it is possible that features of the experimental procedure may have contributed to the effect. The objects on each floor appeared below the horizontal midpoint of the computer screen (arranged so because most objects in the real world are found below eye level); this may have encouraged biased downward visual encoding. When visual memories were relied on in the test, this arrangement might have influenced the test angles systematically, as is suggested by the downward tilt bias on the same floor. Although it is not clear that this would lead to the pattern of effects that we observed, it remains a possibility. Also, the objects on different floors were located at slightly different heights in order to equate, as far as possible, the upward and downward test angles between floors. Again, it does not necessarily follow that this would influence the outcome, but these factors were addressed in Experiment 2.

In Experiment 2, the same procedure was followed as that in Experiment 1, but the VE was modified by adjusting the heights of the stands on which the test objects were located, so that all the objects were raised to appear in the horizontal center of the screen, at subjective "eye level." This ensured that there were no biases in the visual field and that the vertical angles up and down were the same in the test.

\section{Method}

Participants. The participants were 12 1st-year undergraduate students at Leicester University, with a mean age of 20 years and 2 months (range, 20-21 years), who received credit toward a course requirement; 1 participant was male.

Apparatus. The apparatus was the same as that in Experiment 1, except that the stands on which the objects rested were extended vertically upward so that the midpoint of the test objects appeared 

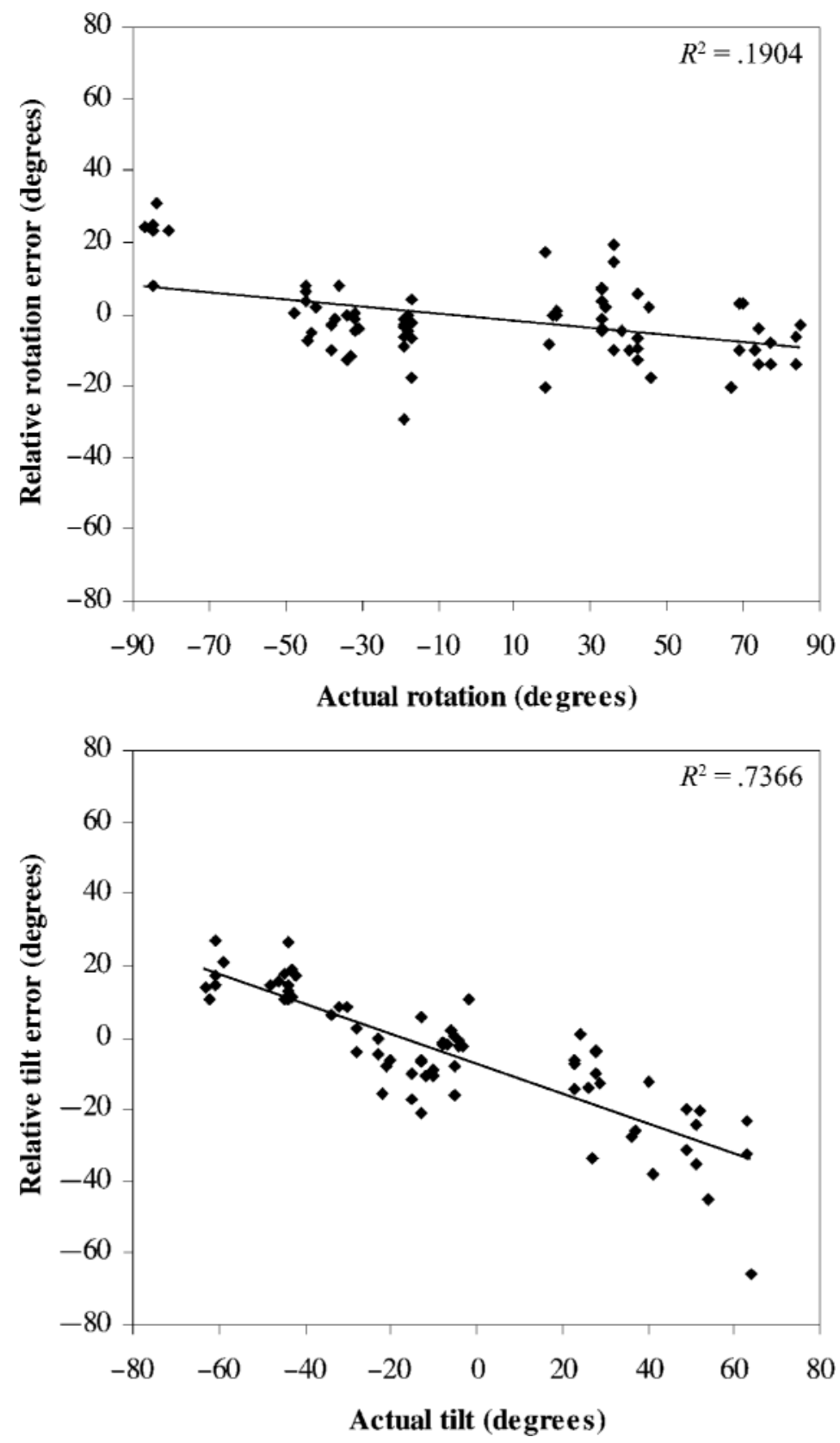

Figure 4. Results of Experiment 1. Scatterplot illustrating the relationship between the actual rotation required to point accurately toward a test object and the participants' error (upper panel) and the relationship between the actual tilt required to point accurately toward a test object and the participants' error (lower panel).

on the horizontal plane midway down the computer screen, or at subjective "eye level."

Procedure. Training and testing were carried out following the procedure in Experiment 1.

\section{Results and Discussion}

The data from this experiment were collated and analyzed in the same way as in Experiment 1. Analyses of the between-conditions variability (see the lower portion of Table 1) suggest that the time and absolute tilt error data were heterogeneous. An examination of the time data suggests that heterogeneity in this case was a consequence of two unusually long observation times from the 12 participants, and little weight should be attached to this result. As in Experiment 1, the absolute tilt analysis suggests more variable representations of the loca- 
tions of objects on higher floors than of those on lower floors or on the same floor.

The mean scores for each floor in the five data sets were analyzed with the nonparametric techniques employed in Experiment 1 for consistency. The mean latency data are illustrated in the top panel of Figure 5. An analysis of these data showed no significant difference between conditions $\left(\chi^{2}=3.2, p>.05\right)$.

The mean relative rotation errors are illustrated in the middle panel of Figure 5. An analysis of these data showed no significant difference between conditions $(p>.1)$. As in Experiment 1, the same-floor data were analyzed separately; no difference was apparent between the number of judgments that tended to the left and the right, with 6 of the 12 participants making judgments to the right (binomial probability $>.1$ ).

The mean relative tilt errors are illustrated in the bottom panel of Figure 5. Negative errors represent errors in which the target was judged as lower than its actual position, whereas positive errors represent errors in which the target was judged as higher than its actual position. Analysis revealed a significant overall difference between floors $\left(\chi^{2}=31.4, p<.05\right)$, with a significant difference between upward and downward judgments $(z=$
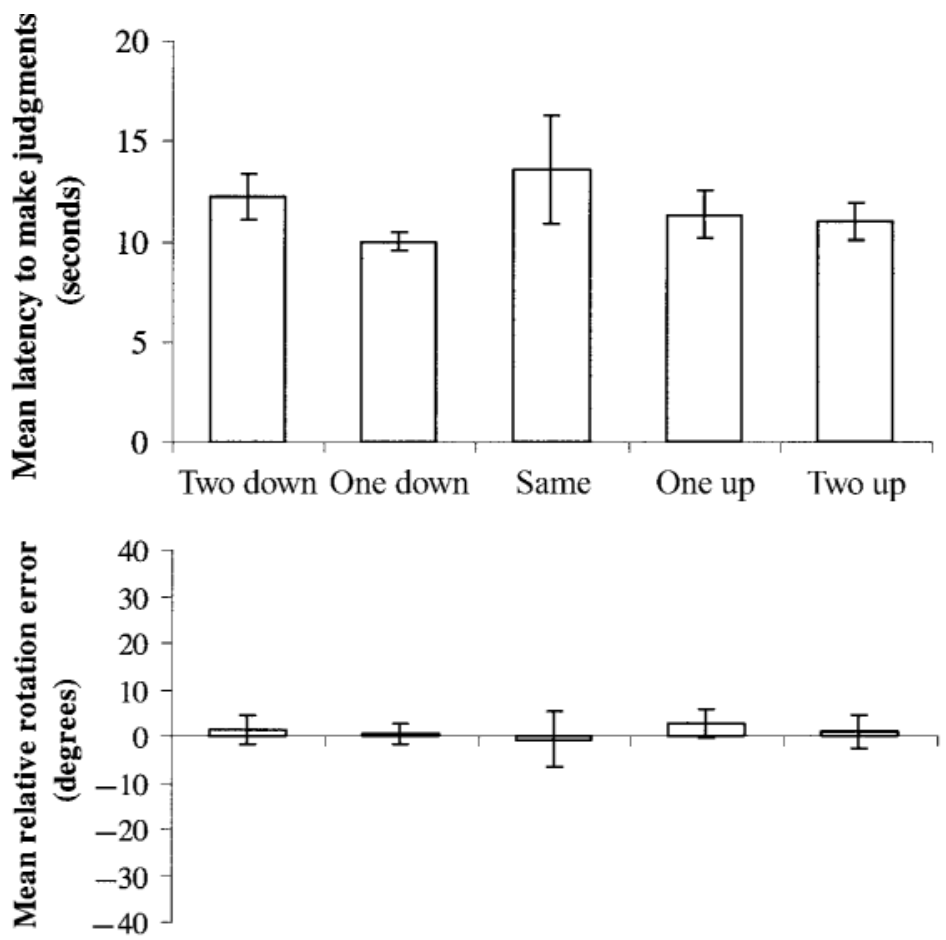

Two down One down Same One up Two up

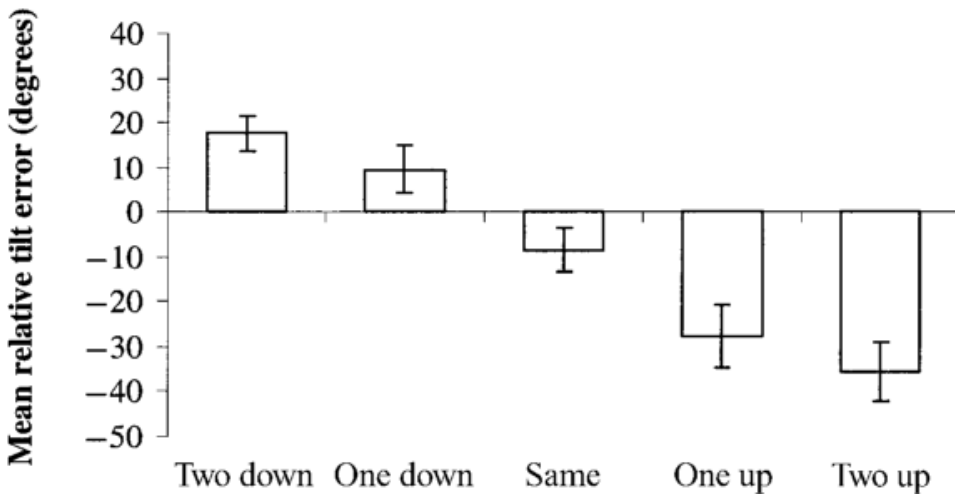

Figure 5. Results of Experiment 2. The top panel illustrates the mean latencies to make the tilt and rotation judgments about the former positions of objects that were two floors up or down, one floor up or down, or on the same floor. The middle panel illustrates the mean relative rotation errors for these judgments. The bottom panel illustrates the mean relative tilt errors for these judgments. The error bars represent one estimated standard error above and below the mean. 
$3.06, p<.05$ ), errors on upward judgments were biased down toward the horizontal plane, whereas errors on downward judgments were biased up toward the horizontal plane. As in Experiment 1, the same-floor data were analyzed separately; 9 of the 12 participants made errors in judgments that were below the horizontal (binomial probability $>.1$ ).

The mean absolute rotation errors can be seen in the top panel of Figure 6 . An analysis revealed a significant overall difference between floors $\left(\chi^{2}=21.4, p<.05\right)$ and a borderline significant difference between upward and downward judgments $(z=1.96, p=.05)$, with greater rotation errors on upward judgments.

The mean absolute tilt errors are illustrated in the bottom panel of Figure 6. Analysis revealed a significant overall difference between floors $\left(\chi^{2}=13.9, p<.05\right)$ with greater errors on upward than on downward judgments $(z=2.04, p<.05)$.

Across the counterbalancing of the positions of objects and test locations on different floors, 42 different rotation measures (range, $84^{\circ}$ to $-86^{\circ}$ ) and 43 different tilt measures (range, $69^{\circ}$ to $-58^{\circ}$ ) were derived. The mean error judgment data for each of these angles were en- tered into correlation analyses as in Experiment 1, which showed a very similar pattern to that found in Experiment 1 . Spearman's rho correlations on the signed tilt and rotation differences yielded a medium negative correlation between the actual degree of rotation and the errors in the participants' relative judgments of rotation $(r=-.35, p<.05$; see the top panel of Figure 7) and a large negative correlation between degree of tilt and participants' relative tilt judgments $(r=-.80, p<.05$; see the bottom panel of Figure 7). As in Experiment 1, both of these correlations reflect errors that increase systematically in their bias toward the center point (directly ahead) as the angle to the objects increased away from the center point, with a greater magnitude of effect for upward than for downward judgments.

The most important finding from Experiment 2 is that, despite the vertical visual symmetry in the positioning of the objects during the learning phase, in the test phase the vertical asymmetry in mean relative and absolute tilt errors and in the asymmetric variance of tilt errors that was found in Experiment 1 was replicated. As in Experiment 1 , the absolute tilt error difference appears to be due largely to the greater relative downward bias on up-
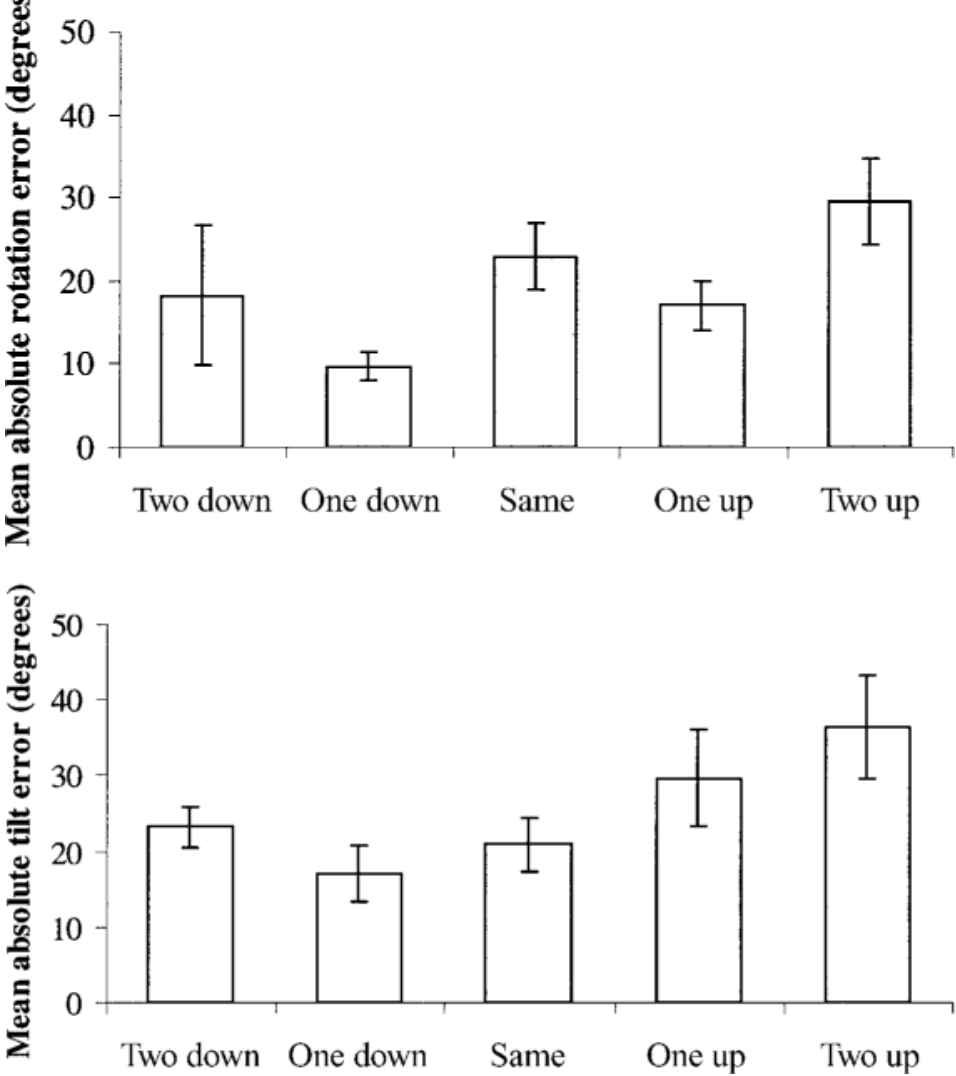

Figure 6. Results of Experiment 2. The top panel illustrates the mean absolute rotation errors for judgments of the former position of objects that were two floors up or down, one floor up or down, or on the same floor. The bottom panel illustrates the mean absolute tilt errors for these judgments. The error bars represent one estimated standard error above and below the mean. 

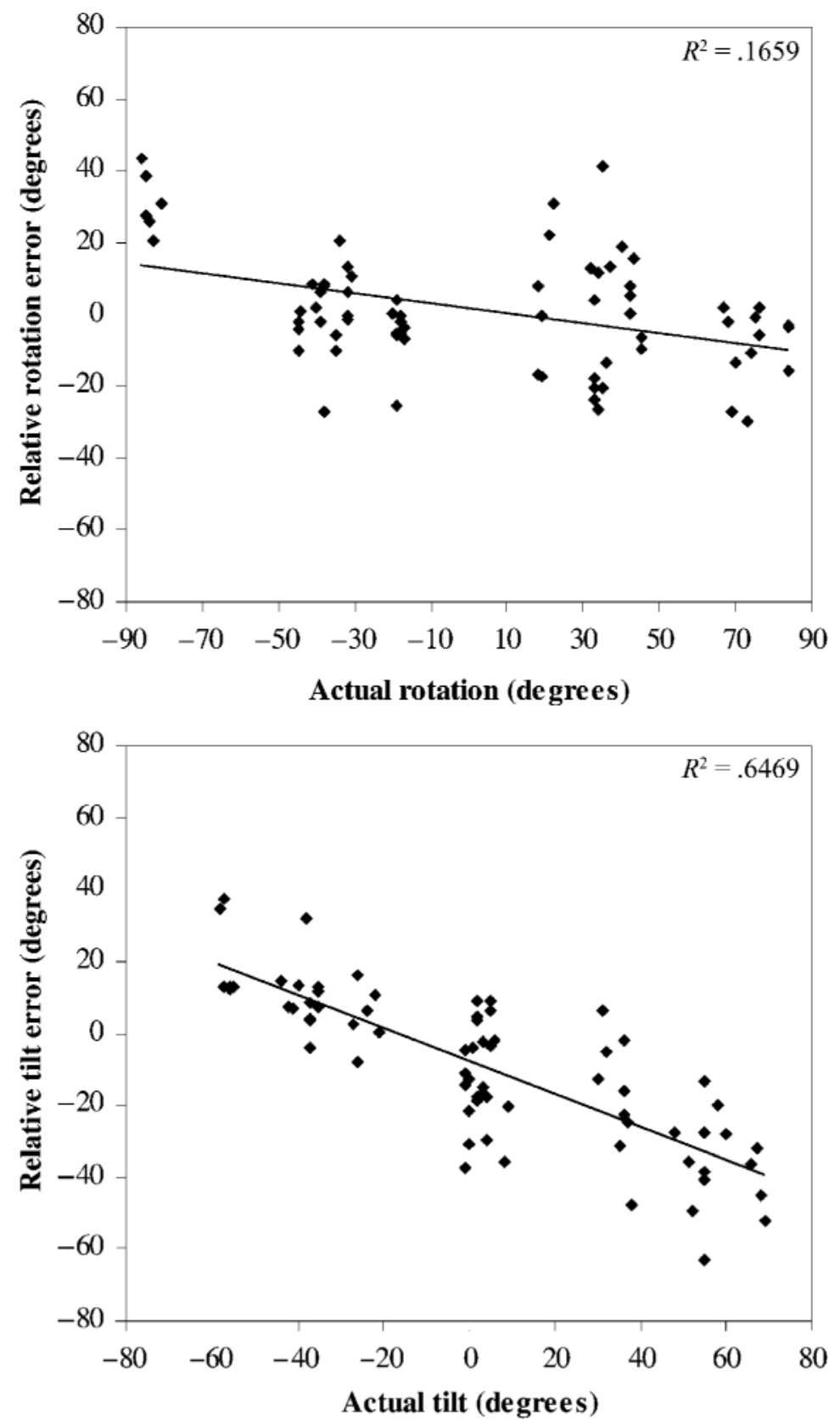

Figure 7. Results of Experiment 2. Scatterplot illustrating the relationship between the actual rotation required to point accurately toward a test object and the participants' error (upper panel) and the relationship between the actual tilt required to point accurately toward a test object and the participants' error (lower panel).

ward judgments $\left(M\right.$ of one and two floors up $\left.=-32^{\circ}\right)$, rather than to the upward bias on downward judgments ( $M$ of one and two floors down $\left.=14^{\circ}\right)$.

On average, judgments were made slightly more quickly in Experiment $2(M=11.6 \mathrm{sec})$ than in Experiment 1 $(M=14.1 \mathrm{sec})$.
Importantly, the outcome of Experiment 2 suggests that the asymmetry in the encoding or retrieval of spatial information cannot be accounted for in terms of vertical asymmetries within the visual field while the particular VE is learned. Of course, vertical asymmetries within the visual field (Aguirre \& D’Esposito, 1997; Prinz, 1984) 
that occur in everyday perception, and prior to the experimental experience, remain a possible influence on spatial encoding within the experiments.

\section{EXPERIMENT 3}

In Experiment 2, presenting objects in the horizontal center of the screen did not substantially alter the outcomes found in Experiment 1. The replication in Experiment 2 of the vertical asymmetry in absolute rotation and tilt errors suggests that vertical asymmetry in spatial memory is not an artifact of the test procedures. The tendency to remember objects as being lower than they were on the same floor in Experiment 1, although not significant in Experiment 2, is particularly interesting and may help to account for the asymmetry in accuracy that characterizes upward and downward judgments. We will return to this in the General Discussion section.

However, there remains one potential explanation of the effects found in Experiments 1 and 2 that does not involve spatial memory: Our accuracy results could be explained if participants were to misestimate the viewpoint height during the test, perceiving it to be lower than its actual height (which was midway between the ceiling and the floor). Such a misperception, when combined with a tendency to underestimate the angles to both upper and lower floors, can lead to a pattern of tilt effects very similar to those reported here. In this case, the actual angle judgments upward and downward could be equally accurate but could present an asymmetric pattern of errors. ${ }^{1}$ Visual inspection of both versions of the VEs used in Experiments 1 and 2 suggests that misperception of the viewpoint height is extremely unlikely; however, given the potential explanatory power of this account, it is necessary to establish empirically that participants do not misperceive the viewing height either during exploration or at test.

\section{Method}

Participants. The participants were 8 undergraduate students at Leicester University; none had any prior experience of the apparatus. Their mean age was 20.9 years, and 2 were men.

Apparatus. The VE used in Experiment 2 was tested. To record judgments of the perceived viewpoint height, an open oblong, $12 \times$ $20 \mathrm{~cm}$, was drawn on a blank sheet of paper to represent a VE room, with the words "ceiling" printed above and "floor" printed below. A 12-cm vertical line was drawn from the ceiling to the floor, $3 \mathrm{~cm}$ in from the left "wall" of the illustration.

Procedure. The participants were informed that they would be asked to watch a short tour through three rooms in a VE and would then be asked to make a judgment about the height that the rooms were seen from. They were told that the viewpoint height with respect to the ceiling and the floor was the same in all three rooms.

The participants sat in front of the screen at the same distance as the participants in Experiments 1 and 2. The experimenter controlled movement through the VE, starting in the middle room, with the viewpoint located in front of the elevator, facing toward the center of the room. This floor was continuously explored for $2 \mathrm{~min}$ from a variety of directions and locations, using forward and backward translations and left and right rotations. Movement to the floor above (or below, counterbalanced) was effected via the elevator, where the same exploration procedure was repeated. Movement to the remaining unexplored floor was again effected via the elevator, and the final exploration was conducted. The final view of the scene remained on the screen while the participants indicated their estimates of the viewpoint height on the prepared illustration sheet by drawing a short line that intersected the single vertical line at the point at which they perceived the viewpoint height to be.

Following this exploration phase, the test views on the VE were presented. Three views were presented, one in each room, with switching between viewpoints effected instantaneously. At each viewpoint, the experimenter rotated the view up and down to ceiling and floor height from a static location, with the initial direction counterbalanced across participants and between floors. The viewpoint height judgment was then repeated on a separate prepared test sheet.

\section{Results}

The distance of the intersecting line that the participants drew was measured from the "ceiling" downward along the $12-\mathrm{cm}$ indicator line; estimates lower than $6 \mathrm{~cm}$ indicate a judged height above the midpoint of the room. Following exploration of the three VE rooms, the participants' mean estimate was $5.7 \mathrm{~cm}(S D=0.96)$; following illustration of the test VE, the mean estimate was $6.1 \mathrm{~cm}(S D=0.86)$. A related $t$ test showed no significant difference between estimates following exploration and estimates following the test site illustration $[t(7)=1.09]$. One-sample $t$ tests comparing each of these judgment scores against a midpoint test value of $6 \mathrm{~cm}$ showed no significant difference in either case $(t s<1)$. There was no suggestion that the participants' judgments deviated from the middle height of the room; therefore, an explanation of the effects found in Experiments 1 and 2 in terms of misperception of the viewpoint height received no support.

\section{GENERAL DISCUSSION}

These experiments are the first, to our knowledge, that have systematically investigated a spatial vertical asymmetry in memories of large-scale space derived from perception. The only other experiments, of which we are aware, that have noted vertical asymmetry are, first, the text-based experiment of Bryant et al. (1992, Experiment 1) and, second, the VE study of Wilson, Foreman, and Tlauka (1997). Bryant et al. found that, after reading text descriptions of spatial relationships, participants were faster to respond from memory to the location of a test object if it was below, rather than above, a referent object. Although other text experiments have shown only small and nonsignificant differences in the same direction, it is possible that the vertical asymmetries evident in this text experiment, and after visual-perceptual learning in the present experiments, are based on similar memory representations. Following VE exploration and testing in a real equivalent environment, Wilson, Foreman, and Tlauka found more accurate absolute rotation judgments on a lower than on a higher floor; on the higher floor, their VE group performed no better than controls. The authors' suggestion, that this result might have arisen 
from a poor representation of the stair area linking ground and upper levels in the VE, remains valid, because the stairways connecting the upper and the lower floors differed. However, the results of the experiments reported in the present article make asymmetrical encoding or retrieval in Wilson, Foreman, and Tlauka's experiment a plausible alternative.

Why is this asymmetry in memory present? It is important to note that there are several biases revealed in the data. First, in Experiment 1, the participants had a tendency to misremember objects as being lower than they actually were when the same-floor judgments were made. Second, they tended to make both upward and downward judgments that were biased toward the horizontal, a regression toward the mean. Third, they made greater downward errors when making upward judgments than upward errors when making downward judgments. Perhaps these effects are interrelated: Given that participants show a downward bias on the same floor, it is reasonable to conclude that they may carry this bias through to judgments on upper and lower floors. A general downward bias would counter a tendency to make vertical errors that are biased toward the horizontal when estimating below the horizontal, but may leave unaffected or even exacerbate errors when estimating above the horizontal. Thus, in principle, much of the present data could be accounted for by relying purely on generalization of the downward bias in spatial memory that is apparent on same-floor judgments. Although we accept this as a reasonable account of the results of Experiment 1 , we have two reservations. First, the downward bias in same-floor judgments was not statistically significant in Experiment 2, but the tilt asymmetry was still evident. Furthermore, in a closely related experimental design, in which both able-bodied children and children with physical disabilities were tested, we have found an asymmetry in judgments from memory that is similar to that reported here, in which upward errors were greater than downward errors (Wilson, Foreman, Stanton, \& Duffy, in press); however, in that experiment, we did not find a downward bias on the same floor. Whether or not the downward bias on same-floor judgments plays a role in the increased accuracy of downward judgments between floors, the results are generally in agreement with the central ideas of the spatial framework analysis (Bryant \& Tversky, 1999; Bryant et al., 1992; Franklin \& Tversky, 1990), in that the effects of gravity not only may lead to the dominance of the head/feet axis, as these authors suggest, but also may lead to more accurate downward spatial processing.

How do our results relate to those in studies in which vertical asymmetry has not been shown? Although Bryant et al. (1992, Experiment 1) found significantly faster responding to objects below a perspective described textually, in other experiments this effect was not significant, and Bryant and Tversky (1999) found nonsignificant differences in the same direction using diagrams and models. An obvious difference between the experiments above and the present series is the medium used to present the spatial information. However, it is interesting to note that in the experiments mentioned above the dependent variable was response time and that, in our experiments, we found no significant evidence of asymmetry in response time measures, but only in directional accuracy and consistency. Therefore, it is possible that these dependent variables reflect different aspects of spatial knowledge. Byrne (1979) has advocated two types of spatial representation: The first type encodes categorical information, which is qualitative information, such as whether an object is above or below an observer, and in which distance and directional information is not encoded, whereas the second type encodes metric or coordinate information, such as relative distance and more precise angles (see also Franklin, 1996; Franklin, Henkel, \& Zangas, 1995; Kosslyn et al., 1989). Similarly, Jackendoff (1987) distinguishes between algebraic and geometric representational levels, which process categorical and metric information, respectively. Rinck, Hähnel, Bower, and Glowalla (1997) have used the term categorical in a related way-to describe regional, rather than directional, categories - and have found that metric information may be acquired but not always used, depending on the test task. Since the present experiments showed a significant vertical asymmetry in pointing accuracy, but not in response times, it may be that the asymmetry occurs in precise spatial localization, rather than in the process of retrieving broad categorical relationships, such as above and below. The present experiments were not designed to address this issue, but the results suggest that this may be a useful direction for future research.

The presence of asymmetry in spatial representations is especially important when considering the potential applications of VE technology for individuals who, for reasons of age, experience, or disability, find it difficult to navigate within large and confusing public buildings, such as shopping malls and museums, and who might benefit from taking virtual tours to acquire information about building layouts and facility locations, in advance of the actual visit (Wilson, Foreman, \& Stanton, 1997). Difficulty in accessing the built environment remains a major factor in limiting the mobility of disabled individuals (Foster, Wenn, Harwin, \& O'Hart, 1998), and thus, educating spatially impaired individuals might, in the future, represent a beneficial application of VE use. Yet large public buildings are typically confusing - specifically, because they consist of multiple levels. The present results suggest that training should be arranged so that additional attention or practice is directed to higher, rather than to lower, locations.

One potential limitation to the generality of our results should be mentioned: We selected opportunity samples for both experiments that, overall, happened to comprise $92 \%$ women, and it may be that the biases we report are largely or partly a result of our predominantly female sample (cf. Astur, Ortiz, \& Sutherland, 1998, who found sex differences in a horizontal VE). Spatial information 
can be acquired in many forms, with alternative strategies and environmental cues (see Foreman \& Gillett, 1997). Moreover, individual differences among participants, including gender, have been shown to have modest effects on the type and quality of information obtained in real environments and in VEs (Moffat, Hampson, \& Hatzipantelis, 1998; Waller, 2000). However, in many studies, no sex differences have been found (e.g., Rossano et al., 1999). Such differences are probably marginal, as compared with the great improvements on chance performance and substantial reductions in angular pointing errors that occur after virtual training. Darken and Sibert (1996) reported that where training advantages were evident, these were not gender specific. Of more direct relevance to the present study is our recent investigation of whether children (both able-bodied and disabled) also show a vertical asymmetry similar to that found in the present experiments (Wilson et al., in press). We found that relative and absolute tilt errors followed a pattern similar to those described in the present experiments; however, despite a slightly greater number of male than female participants, we found no differences on any measure due to the sex of the participants.

In summary, participants who explored a threedimensional computer simulation of a multistory VE were found to be more accurate and consistent when judging the relative position of remembered test objects that were located below the horizontal test plane. This novel finding suggests an important addition to our understanding of the nature of spatial representations. Furthermore, there are potential practical implications for learning about spatial arrays on more than one level that need to be kept in mind when VEs are used in navigational spatial training. We are currently investigating whether the asymmetry that we have found in VEs occurs also following real-world exploration.

\section{REFERENCES}

Aguirre, G. K., \& D’Esposito, M. (1997). Environmental knowledge is subserved by separable dorsal/ventral neural areas. Journal of Neuroscience, 17, 2512-2518.

Astur, R. S., Ortiz, M. L., \& Sutherland, R. J. (1998). A characterisation of performance by men and women in a virtual Morris water task: A large and reliable sex difference. Behavioural Brain Research, 93, 185-190.

BRyANT, D. J., \& TVERSKy, B. (1999). Mental representations of perspective and spatial relations from diagrams and models. Journal of Experimental Psychology: Learning, Memory, \& Cognition, 25, 137-156.

Bryant, D. J., Tversky, B., \& Franklin, N. (1992). Internal and external spatial frameworks for representing described scenes. Journal of Memory \& Language, 31, 74-98.

BYRNE, R. W. (1979). Memory for urban geography. Quarterly Journal of Experimental Psychology, 31A, 147-154.

DARKEN, R. P., \& SibERT, J. L. (1996). Navigating large virtual spaces. International Journal of Human-Computer Interaction, 8, 49-71.

FOREMAN, N., \& GILLETT, R. (1997). Handbook of spatial research paradigms and methodologies: Vol. 1. Spatial cognition in the adult and child. Hove, U.K.: Psychology Press.

Foreman, N., Stanton, D., Wilson, P. N., \& Duffy, H. (2003). Spatial knowledge of a real school environment acquired from virtual or physical models by able-bodied children and children with disabilities. Journal of Experimental Psychology: Applied, 9, 67-74.

Foster, G. T., Wenn, D. E. N., Harwin, W. S., \& O'Hart, F. (1998). Generating virtual environments to allow increased access to the built environment. International Journal of Virtual Reality, 3, 12-20.

FRANKLIN, N. (1996). Language as a means of constructing and conveying cognitive maps. In J. Portugali (Ed.), The construction of cognitive maps (pp. 275-295). Dordrecht: Kluwer.

FrankLIN, N., HENKEL, L. A., \& ZANGAS, T. (1995). Parsing surrounding space into regions. Memory \& Cognition, 23, 397-407.

FRANKLIN, N., \& TVERSKY, B. (1990). Searching imagined environments. Journal of Experimental Psychology: General, 119, 63-76.

Genzano, V. R., Di Nocera, F., \& Ferlazzo, F. (2001). Upper/lower visual field asymmetry on a spatial relocation memory task. NeuroReport, 12, 1227-1230.

JACKENDOFF, R. (1987). Consciousness and the computational mind. Cambridge, MA: MIT Press.

Kosslyn, S. M., Koenig, O., Barrett, A., Cave, C. B., Tang, J., \& GABRIELI, J. D. E. (1989). Evidence for two types of spatial representations: Hemispheric specialization for categorical and coordinate relations. Journal of Experimental Psychology: Human Perception \& Performance, 15, 723-735.

KuiPers, B. (1978). Modelling spatial knowledge. Cognitive Science, 2 , $129-153$.

Ladavas, E., CARletti, M., \& Gori, G. (1994). Automatic and voluntary orienting of attention in patients with visual neglect: Horizontal and vertical dimensions. Neuropsychologia, 32, 1195-1208.

MofFAT, S. D., HAMPSON, E., \& HATZIPANTELIS, M. (1998). Navigation in a "virtual" maze: Sex differences and correlation with psychometric measures of spatial ability in humans. Evolution \& Human Behavior, 19, 73-87.

PÉRUCH, P., \& GAUNET, F. (1998). Virtual environments as a promising tool for investigating human spatial cognition. Current Psychology of Cognition, 17, 881-899.

PÉruCh, P., VerCher, J. L., \& GAUthier, G. M. (1995). Acquisition of spatial knowledge through visual exploration of simulated environments. Ecological Psychology, 7, 1-20.

PoucE T, B. (1993). Spatial cognitive maps in animals: New hypotheses on their structure and neural mechanisms. Psychological Review, 100, 163-182.

Pre VIC, F. H. (1990). Functional specialization in the lower and upper visual fields in humans: Its ecological origins and neurophysiological implications. Behavioral \& Brain Sciences, 13, 519-575.

Pre vic, F. H. (1998). The neuropsychology of 3-D space. Psychological Bulletin, 124, 123-164.

Prinz, W. (1984). Attention and sensitivity in visual search. Psychological Review, 45, 355-366.

Regian, J. W., Shebilske, W. L., \& Monk, J. M. (1992). Virtual reality: An instructional medium for visual-spatial tasks. Journal of Communication, 4, 136-149.

Richardson, A. E., Montello, D. R., \& Hegarty, M. (1999). Spatial knowledge acquisition from maps and from navigation in real and virtual environments. Memory \& Cognition, 27, 741-750.

Rinck, M., HäHNel, A., Bower, G. H., \& Glowalla, U. (1997). The metrics of spatial situation models. Journal of Experimental Psychology: Learning, Memory, \& Cognition, 23, 622-637.

Rose, F. D., \& Foreman, N. P. (1999). Virtual reality. The Psychologist, 12, 550-554.

Rossano, M. J., West, S. O., Robertson, T. J., Wayne, M. C., \& CHASE, R. B. (1999). The acquisition of route and survey knowledge from computer models. Journal of Environmental Psychology, 19, 101-115.

Rubin, N., NAKayama, K., \& Shapley, R. (1996). Enhanced perception of illusory contours in the lower versus upper visual hemifields. Science, 271, 651-653.

Shelton, A. L., \& GABrieli, J. D. E. (2002). Neural correlates of encoding space from route and survey perspectives. Journal of Neuroscience, 22, 2711-2717.

Stanton, D., Wilson, P. N., \& Foreman, N. (1996). Using virtual reality environments to aid spatial awareness in disabled children. In 
P. M. Sharkey (Ed.), Proceedings of the 1st European Conference on Disability, Virtual Reality and Associated Technologies (pp. 93-101). Reading, U.K.: University of Reading.

WALLER, D. I. (2000). Individual differences in spatial learning from computer-generated environments. Journal of Experimental Psychology: Applied, 6, 307-321.

WiLSON, P. N. (1997). Use of virtual reality computing in spatial learning research. In N. Foreman \& R. Gillett (Eds.), Handbook of spatial research paradigms and methodologies: Vol. 1. Spatial cognition in the adult and child (pp. 181-206). Hove, U.K.: Psychology Press.

Wilson, P. N., Foreman, N., \& STANTON, D. (1997). Virtual reality, disability and rehabilitation. Disability \& Rehabilitation, 19, 213 220.

Wilson, P. N., Foreman, N., Stanton, D., \& Duffy, H. (in press). Memory for targets in a multi-level simulated-environment: A com- parison between able-bodied and disabled children. British Journal of Psychology.

Wilson, P. N., Foreman, N., \& Tlauka, M. (1997). Transfer of spatial information from a virtual to a real environment. Human Factors, 39, 526-531.

Witmer, B. G., Bailey, J. H., Knerr, B. W., \& Parsons, K. C. (1996). Virtual spaces and real world places: Transfer of route knowledge. International Journal of Human-Computer Studies, 45, 413-428.

\section{NOTE}

1. We are grateful to an anonymous reviewer for this insight.

(Manuscript received June 21, 2002;

revision accepted for publication September 8, 2003.) 